\title{
High Expression of EIF4G2 Mediated By the TUG1/hsa-miR- 26a-5p Axis Is Associated With Poor Prognosis and Immune Infiltration of Gastric Cancer
}

Liu Fu

Tongji University

Zhe Wang

Tongji University School of Medicine

Fengxiang Jiang

Tongji University

Guohua Wei

Tongji University

Longe Sun

Tongji University

Chuanyong Guo

Tongji University

Jianye Wu

Tongji University

Jianhuan Zhu ( $\nabla$ wjymail@163.com )

Tongji University

Research Article

Keywords: EIF4G2, gastric cancer, hsa-miR-26a-5p, TUG1, prognosis, immune infiltration

Posted Date: December 3rd, 2021

DOI: https://doi.org/10.21203/rs.3.rs-1107778/v1

License: (c) (i) This work is licensed under a Creative Commons Attribution 4.0 International License. Read Full License 


\section{Abstract}

Background: Eukaryotic translation initiation factor 4 gamma 2 (EIF4G2) is involved in the occurrence and development of various tumors. However, the effect of EIF4G2 in gastric cancer (GC) has not been fully explored. The purpose of this study was to explore the function and mechanism of EIF4G2 in GC.

Methods: The Tumor Immune Estimation Resource 2.0 database was used to analyze EIF4G2 expression in various cancers and the relationship between EIF4G2 expression and tumor-infiltrating immune cells. Gene Expression Profiling Interactive Analysis was utilized to assess the EIF4G2 expression level and its effect on survival in GC. UALCAN was conducted to analyze EIF4G2 expression in various sub-groups of GC. The Kaplan-Meier plotter was employed for survival analysis. Receiver Operator Characteristic (ROC) curve analysis was applied to evaluate the diagnostic role of EIF4G2 in GC. LinkedOmics was used to identify the co-expressed genes and Gene Ontology and Kyoto Encyclopedia of Genes and Genomes pathways. The Tumor-Immune System Interaction database was employed to analyze the correlation between EIF4G2 expression and tumorinfiltrating lymphocytes. The starBase web platform was used to predict the upstream microRNAs and long non-coding RNAs.

Results: EIF4G2 expression was upregulated in GC tissues compared to normal controls. High expression of EIF4G2 indicated poor prognosis in GC. ROC analysis revealed that EIF4G2 had good diagnostic ability to distinguish GC from normal tissues. Immune infiltration analysis indicated that EIF4G2 expression may be involved in the modulation of tumor immune infiltration in GC. Finally, we determined that the Taurine Up-Regulated 1 (TUG1)/hsa-miR-26a-5p/EIF4G2 axis was the most likely regulatory pathway involved in GC development.

Conclusions: EIF4G2 was upregulated in GC and elevated expression of EIF4G2 indicated unfavorable prognosis. Moreover, EIF4G2 expression may be involved in the regulation of tumor immune cell infiltration. The TUG1/hsa-miR-26a-5p axis is a likely upstream regulatory mechanism of EIF4G2 in GC. EIF4G2 may thus serve as a prognosis biomarker and present a new therapeutic target.

\section{Background}

Gastric cancer (GC) is a serious threat to human health worldwide, which ranks fifth in incidence and fourth in mortality among all cancers[1, 2]. Since the early clinical symptoms are not obvious, most GC patients are already in the advanced stage when diagnosed[3]. Therapeutic methods are few and their effectiveness is limited to later-period tumor patients, when the 5-year survival rate is poor $[4,5]$. Therefore, it is necessary to explore the molecular mechanisms that regulate GC occurrence and develop novel biomarkers for early diagnosis.

The function of eukaryotic translation initiation factors (elFs) in tumorigenesis has been widely studied. Studies have shown that elFs are highly expressed in many malignant tumors[6-8]. Eukaryotic translation initiation factor 4 gamma 2 (EIF4G2) is a member of the elF family, which plays a vital role in regulating protein translation[9-11]. Research has revealed that abnormal expression of EIF4G2 is closely related to the occurrence and development of tumors. Some research has suggested EIF4G2 acts as oncogene. For example, downregulation of EIF4G2 by microRNA (miR)-379 inhibits the proliferation, migration and invasion of osteosarcoma cells[12]. Diffuse large B-cell lymphoma (DLBCL) cell lines and patient samples have shown upregulated EIF4G2 expression, while downregulation of EIF4G2 decreased translation, cell proliferation and cell colony formation, thus inhibiting DLBCL development[13]. However, another study found that EIF4G2 transcripts were downregulated in bladder tumors, and this downregulation was associated with invasive tumors[14]. In GC, the effect of EIF4G2 has not been fully explored.

In the present study, various public online databases were utilized to analyze EIF4G2 expression. Firstly, Tumor Immune Estimation Resource 2.0 (TIMER2.0), Gene Expression Profiling Interactive Analysis (GEPIA), UALCAN databases and the Kaplan-Meier plotter were employed to analyze the expression level and prognosis of EIF4G2 in GC. Then, the co-expression of genes and their relationship with immune cells were explored using LinkedOmics, TIMER2.0 and the Tumor-Immune System Interaction database (TISIDB). Finally, the starBase web platform was used to predict upstream microRNAs (miRNAs) and long non-coding RNAs (IncRNAs). In our study, we found that EIF4G2 was upregulated in GC, and high expression of EIF4G2 
indicated poor prognosis. Moreover, EIF4G2 participated in the regulation of tumor immune cell infiltration in GC. The Taurine Up-Regulated 1 (TUG1)/hsa-miR-26a-5p axis was determined to be the most likely upstream regulatory mechanism for EIF4G2 in GC.

\section{Methods}

\section{TIMER2.0 database}

TIMER2.0( http://timer.cistrome.org/ ) is a web server for the systematic analysis of tumor-infiltrating immune cells (TIICs) across diverse cancer types[15]. It allows users to compare the expression of a gene between tumor and normal tissues in multiple cancers. In this study, the TIMER2.0 website was used to analyze differential expression of EIF4G2 in tumor and normal tissues in various cancers. We then predicted the relationship between EIF4G2 expression in GC and six tumorinfiltrating immune cells, including B cells, CD4+ T cells, CD8+ T cells, neutrophils, macrophages and dendritic cells (DCs). In addition, we analyzed the correlation between the molecular markers of immune cells and expression of EIF4G2.

\section{GEPIA database}

GEPIA( http://gepia.cancer-pku.cn/ ) is an interactive online web tool for the analysis of tissue samples from The Cancer Genome Atlas (TCGA) and the Genotype-Tissue Expression (GTEx) projects[16]. In this study, the website was used to explore mRNA expression of EIF4G2 in tumor and normal tissues. Also, we determined the association between EIF4G2 expression and overall survival of patients with GC.

\section{UALCAN database}

UALCAN( http://ualcan.path.uab.edu/ )is a comprehensive web portal to perform in-depth analyses of TCGA gene expression data[17]. In this study, UALCAN was employed to explore the mRNA expression of EIF4G2 across tumor and normal samples, as well as in various tumor sub-groups based on patients' gender, nodal metastasis status, individual cancer stage and tumor grade.

\section{Kaplan-Meier plotter (KM plotter) database}

The KM plotter( http://kmplot.com/analysis/index.php?p=background ) is a meta-analysis-based platform for survival analysis of $54 \mathrm{k}$ genes (mRNA, miRNA and protein-coding) in 21 types of cancers, the data sources of which include the Gene Expression Omnibus (GEO), European Genome-phenome Archive and TCGA[18]. In this study, the KM plotter was applied to evaluate the relationship between clinical outcomes and EIF4G2 expression in GC. The association between has-miR-26a expression and cancer survival was also evaluated using this web database.

\section{Receiver Operating Characteristic (ROC) curve}

The diagnostic role of EIF4G2 in GC was evaluated by ROC curve analysis based on RNA sequencing (RNA-Seq) data in the transcripts per million (TPM) format of TCGA_GTEx, which were downloaded from UCSC XENA ( https://xenabrowser.net/datapages/ ). R software (version 3.6.3) was used for analysis and visualization.

\section{LinkedOmics database}

LinkedOmics ( http://www.linkedomics.org/login.php) is a publicly available portal that includes multi-omics data from all 32 TCGA cancer types and ten Clinical Proteomics Tumor Analysis Consortium cancer cohorts[19]. In this study, the LinkFinder module of LinkedOmics was used to identify the co-expressed genes of EIF4G2 in GC and produce volcano plots and related heat maps. The co-expressed genes were then used for Gene Ontology (GO) and Kyoto Encyclopedia of Genes and Genomes (KEGG) pathway analysis in the Linklnterpreter module.

\section{TISIDB database}


TISIDB ( http://cis.hku.hk/TISIDB/ ) is an online web portal for examining tumor and immune system interactions[20]. In this study, we use the TISIDB to analyze the correlation between EIF4G2 expression and tumor-infiltrating lymphocytes (TILs) across human cancers. Based on the gene expression profile, the relative abundance of TILs was inferred by using gene set variation analysis. The correlations between EIF4G2 and TILs were measured by Spearman's test.

\section{StarBase database analysis}

StarBase( https://starbase.sysu.edu.cn/ ) is an open-source platform for exploring the miRNA-RNA, RNA-RNA and protein-RNA interactions from UV cross-linking and immunoprecipitation (CLIP)-seq, degradome-seq and RNA-RNA interactome data[21]. In this study, the online tool was used to predict miRNAs binding to EIF4G2. The miRNAs that were identified by more than two programs (PITA, RNA22, miRmap, microT, miRanda, PicTar and TargetScan) were selected for subsequent analyses. The candidate IncRNAs were also explored by using the platform. In addition, starBase was employed to perform expression correlation analysis for miRNA-EIF4G2, IncRNA-miRNA and IncRNA-EIF4G2 interactions in GC. The expression level of miRNAs and IncRNAs in tumor and normal samples was also analyzed in GC by starBase.

\section{RNA-Seq data of TUG1 in GC}

The RNA-Seq data of TUG1 for expression level and survival analysis in GC were obtained from TCGA. For further study, RNAseq data with Fragments Per Kilobase per Million type format were converted to TPM format and subjected to log2 transformation. R software (version 3.6.3) was used for analysis and visualization.

\section{Statistical analysis}

Student's $t$-test was used to compare the gene expression level in tumor and normal samples. Survival analysis was conducted using log-rank tests. ROC curve analysis was applied to detect the cutoff value of EIF4G2 using the pROC package (version 1.17.0.1). $P<0.05$ was considered statistically significant in this study.

\section{Results}

\section{EIF4G2 was upregulated in GC}

Firstly, we analyzed the expression levels of EIF4G2 in multiple tumors and normal tissues based on the TIMER2.0 database. As shown in Figure 1A, compared with normal controls, EIF4G2 was markedly upregulated in a variety of cancers, including esophageal carcinoma (ESCA), cholangiocarcinoma (CHOL), glioblastoma multiforme (GBM), head and neck squamous cell carcinoma (HNSC), liver hepatocellular carcinoma (LIHC), lung squamous cell carcinoma (LUSC) and stomach adenocarcinoma (STAD). Then, the GEPIA database was used to validate the expression of EIF4G2. The results showed that EIF4G2 expression was significantly elevated in GC (Figure 1B, P<0.01). Subsequently, the association between EIF4G2 expression and clinicopathological features in GC patients was investigated using the UALCAN platform. As presented in Figure $1 \mathrm{C}-1 \mathrm{G}$, we found that based on the analysis of sample types, patients' gender, nodal metastasis status, individual cancer stages and tumor grades, the expression of EIF4G2 in GC tissues was significantly higher than in normal tissues. All the above suggested that EIF4G2 was abnormally over-expressed in GC and could possibly serve as a biomarker of GC.

\section{High expression of EIF4G2 indicated poor prognosis in GC}

Next, survival analysis was performed to predict whether the expression of EIF4G2 affected GC patients' prognoses. As depicted in Figure 2A, higher mRNA levels of EIF4G2 in GC were significantly associated with shorter overall survival (OS) in GEPIA. Then, the Kaplan-Meier plotter database was used to evaluate the prognosis associated with EIF4G2 expression in patients with GC, as well as the prognosis in different pathological subtypes. Figure 2B-2D shows that high expression of EIF4G2 was significantly correlated with poor OS, post progression survival (PPS) and first progression (FP) in GC. Exploiting the RNA-Seq data, we further verified the effect of EIF4G2 on the survival of GC patients. The results showed that high expression of EIF4G2 was negatively correlated with OS in patients with grade 3 , stage 2 and stage 4 cancers (Figures $2 \mathrm{E}-2 \mathrm{H}, \mathrm{P}<0.05$ ). Finally, in view of its prognostic value in GC, we generated ROC curves to further analyze the diagnostic value of EIF4G2 in GC. As shown 
in Figure $2 \mathrm{l}$, the area under the curve (AUC) value was 0.844 , indicating that EIF4G2 had good diagnostic ability to distinguish $\mathrm{GC}$ from normal controls. These results indicate that high expression of EIF4G2 is a biomarker of poor prognosis in GC, and that EIF4G2 may serve as a diagnostic biomarker for GC.

\section{EIF4G2 co-expression network in GC}

To investigate the mechanism of action for EIF4G2, the co-expression network of EIF4G2 was constructed using the LinkedOmics database. A volcano plot indicated that 11954 genes (dark red dots) were positively correlated with EIF4G2 expression, and 8271 genes (dark green dots) were negatively correlated (Figure $3 \mathrm{~A}$ ). The 50 genes with the strongest positive and negative correlations are presented in Figure 3B-3C. Gene set enrichment analysis was then applied to analyze the GO terms and KEGG pathways of the genes co-expressed with EIF4G2. The results showed that at the GO_BP (biological process) level, these genes were mainly enriched in cargo loading into vesicle (Figure 3D). GO_ CC (cellular component) was mainly involved in endoplasmic reticulum exit site, coated membrane and chromosomal region, among others (Figure 3E). GO_MF (molecular function) was mainly related to ubiquitinyl hydrolase activity, helicase activity, structural constituent of nuclear pore and regulatory RNA binding, among others (Figure 3F). KEGG pathway analysis indicated that the genes joined mainly in ubiquitin-mediated proteolysis, circadian rhythm, inositol phosphate metabolism, TGF-beta signaling pathway and phosphatidylinositol signaling system (Figure 3G).

\section{Correlation analysis between EIF4G2 expression and immune cell infiltration}

\section{in GC}

Using TIMER 2.0, we analyzed the correlation between EIF4G2 expression and the six types of TIICs. As shown in Figure 4A, EIF4G2 expression was significantly and positively associated with CD8+ T cells $(r=0.257, P=3.74 e-07)$, neutrophils $(r=0.283$, $P=1.99 \mathrm{e}-08)$, macrophages $(r=0.288, P=1.19 \mathrm{e}-08)$ and $D C s(r=0.138, P=7.00 \mathrm{e}-03)$ in GC. However, the results showed a negative correlation with infiltrating levels of $B$ cells $(r=-0.114, P=2.68 \mathrm{e}-02)$ and no correlation with CD4+ T cells $(r=0.026, P$ $=6.10 \mathrm{e}-01$ ). Then, we further evaluated the correlation between EIF4G2 expression and 28 types of TILs in the TISIDB database. Figure 4B shows the relationship between expression of EIF4G2 and 28 types of TILs across human cancers. As presented in Figure 4C-4F, the expression of EIF4G2 was correlated with abundance of Type 2 T helper cells $(T h 2 ; r=0.196, P=6.17 e-05)$, activated CD4 T cells (Act_CD4; $r=0.163, P=0.00086$ ), effector memory CD4 T cells (Tem_CD4; $r=0.164, P=0.000832)$, and immature DCs (iDCs; $r=0.122, P=0.013$ ). These data indicated that EIF4G2 may play a specific role in immune infiltration in GC.

\section{Correlation between EIF4G2 expression and immune cell markers in GC}

Next, we further explored the relationship between EIF4G2 expression and TIIC markers in GC using the TIMER database. As shown in Table 1, we found that EIF4G2 was positively correlated with B cell markers (CD38), CD4 T Cell markers (CD4), M1 macrophage markers (NOS2, IRF5, PTGS2), M2 macrophage markers (CD163, VSIG4, MS4A4A, ARG1, MRC1), neutrophil markers (CEACAM8, ITGAM, CCR7, MPO), DC markers (NRP1, ITGAX, CD141), monocyte markers (CSF1R, CD86), natural killer cell markers (KIR2DS4, KIR3DL2, KIR3DL1, KIR2DL4, KIR2DL3, KIR2DL1), T cell markers (CD2), T cell exhaustion markers (CTLA4, HAVCR2), tumor-associated macrophage (TAM) markers (IL10), T follicular helper (Tfh) markers (BCL6, IL21), Th1 markers (TBX21, STAT4, IFNG), Th2 markers (STAT6, STAT5A), Th17 markers (STAT3) and T regulatory (Treg) markers (FOXP3, CCR8, STAT5B, TGFB1). The results showed that EIF4G2 could be involved in the regulation of tumor immune infiltration in GC.

\section{Prediction and analysis of upstream miRNAs of EIF4G2}

It has been widely acknowledged that non-coding (nc)RNAs participate in the regulation of gene expression. To ascertain whether EIF4G2 was modulated by some ncRNAs, we first predicted upstream miRNAs that could potentially bind to EIF4G2. Considering the underlying mechanisms of miRNAs in the regulation of target gene expression, we predicted a negative correlation between miRNAs and EIF4G2. Finally, we found 15 negatively correlated miRNAs $($ Table $2, P<0.05)$ by starBase. Then, the expression level of these 15 miRNAs in GC and normal samples were examined. The miRNAs with low and 
statistically significant expression levels in GC patients were considered for analysis. Eventually, hsa-miR-26a-5p was identified. As shown in Figure 5A, EIF4G2 was negatively correlated with hsa-miR-26a-5p, and only hsa-miR-26a-5p was significantly downregulated in GC (Figure 5B, P < 0.001). Subsequently, the prognostic value of hsa-miR-26a-5p in GC was investigated. As described in Figure $5 \mathrm{C}-5 \mathrm{~F}$, upregulation of hsa-miR-26a-5p was positively correlated with OS and with favorable OS in patients with stage 2, stage 4 and grade 3 cancers. These findings all suggested that hsa-miR-26a-5p may be the most likely regulatory miRNA of EIF4G2 in GC.

\section{Prediction and analysis of upstream IncRNAs of EIF4G2}

Next, the upstream IncRNAs of hsa-miR-26a-5p were predicted using the starBase database. According to the competing endogenous RNA (ceRNA) hypothesis, IncRNAs can increase mRNA expression by competitively binding to shared inhibitory miRNAs. Therefore, there should be negative correlation between IncRNAs and miRNAs and a positive correlation between IncRNAs and mRNAs. A total of nine possible IncRNAs were predicted (Table 3-4, $\mathrm{P}<0.05$ ). Then, the expression levels and prognostic values of these IncRNAs in GC were assessed. The IncRNAs with high and statistically significant expression levels and unfavorable prognosis in GC patients were further analyzed. Ultimately, only TUG1 met the requirements. Figure 6A-6B shows the negative correlation between hsa-miR-26a-5p and TUG1 and the positive correlation between TUG1 and EIF4G2. As shown in Figure 6C-6D, TUG1 was significantly upregulated in GC compared with normal controls. As described in Figure 6E-6I, overexpressed TUG1 indicated poor OS or shorter disease-specific survival (DSS) of GC patients with different clinicopathological parameters. Taking expression analysis, survival analysis and correlation analysis into consideration, TUG1 appears to be the most likely upstream IncRNA regulating the hsa-miR-26a-5p/EIF4G2 axis in GC.

\section{Discussion}

GC is a malignant tumor with high incidence and poor prognosis worldwide. Exploring the molecular mechanism of GC tumorigenesis may provide a new direction for identifying potential therapeutic targets and prognostic biomarkers. ElF4F is known as a protein complex which functions to regulate translation, composed of the cap-binding protein EIF4E, scaffolding protein EIF4G and ATP-dependent RNA helicase EIF4A[22]. EIF4G2 (alias: death associated protein 5, DAP5; p97) is a subtype of EIF4G[23]. Many studies have reported that EIF4G2 is abnormally expressed in different cancers and has great influence on the progression of tumors. For instance, miR-139-5p suppresses aberrant protein translation by downregulating EIF4G2 expression in myeloid leukemia[24]. Leukemia/lymphoma-related factor interacts with DAP5 to inhibit p53 expression, resulting in tumor cell growth in colon cancer[25]. A recent study found that LINC01579 promotes cell proliferation by competitively binding with miR-139-5p to upregulate EIF4G2 in glioblastoma[26]. Another discovery confirmed that EIF4G2 is upregulated in HCC tissues, and high expression of EIF4G2 has been strongly correlated with worse prognosis in tumor patients[27]. However, the expression level and effect of EIF4G2 in GC are still unknown and deserve further investigation.

In our research, we first used multiple databases to analyze the expression level, survival and prognosis of EIF4G2 in GC. The analysis showed that EIF4G2 was markedly upregulated in GC compared with normal controls. EIF4G2 expression in GC patients with different clinicopathological features revealed that the expression level was higher in patients with advanced tumors. Further survival analysis found that a high expression level of EIF4G2 was significantly correlated with poor OS, PPS and FP in GC. Moreover, the increasing expression of EIF4G2 in higher tumor stages and grades also indicated shorter OS. ROC curves indicated that EIF4G2 had good diagnostic ability to distinguish GC from normal controls. Taken together, the results demonstrated that EIF4G2 may play a key role in the carcinogenesis and progression of GC and should be regarded as a prognostic and diagnostic biomarker.

We then developed a co-expression network for EIF4G2 in GC to explore its underlying mechanisms of action. The genes that positively or negatively correlated with EIF4G2 expression are shown in Figure 3. Some of these genes have been reported to be involved in the development and prognosis of GC. For example, a study suggested that COPI coat complex subunit beta 1 (COPB1) mRNA level was upregulated in GC and indicated poor OS, while COPB1 expression in GC was positively correlated with TILs, PD-L1 and CTLA4[28]. Another study revealed that miR-204-5p inhibited GC cell proliferation via downregulation of USP47 and RAB22A[29]. Histone deacetylase 10, a gene negatively associated with EIF4G2 expression, was found to be

Page 6/22 
downregulated in GC and was associated with an unfavorable prognosis[30]. Therefore, these findings further validated that the function of EIF4G2 in GC might partly involve interactions with these genes.

Next, through the database analysis, we observed that EIF4G2 expression was involved in tumor immune infiltration, and was positively associated with CD8+ T cell, neutrophil, macrophage and dendritic cell infiltration levels. Additionally, the expression of EIF4G2 exhibited positive correlations with most biomarkers of TIICs, including those for macrophages, neutrophils, DCs, TAMs and different T cell subsets. High infiltration of macrophages or TAMs has been reported to be associated with invasion and metastasis of GC, causing poor prognosis[31, 32]. Also, studies suggested that neutrophils could promote GC cell migration[33,34]. Numerous reports about different functional T cell subsets have revealed that these immune cells contribute to tumor development and worse clinical outcomes[35-37]. These findings further confirm that EIF4G2 expression may be involved in the modulation of tumor immune infiltration in GC and influence the progression and prognosis of tumors.

An increasing number of studies have confirmed that ncRNAs such as miRNAs and IncRNAs are involved in the regulation of gene expression according to the ceRNA mechanism, in which IncRNA is regarded as an "miRNA sponge" that binds to inhibitory miRNAs targeting potential mRNAs and prevents their interaction with the mRNA, thus elevating the expression level of the targeted gene and in this way participate in the development of tumors[38-42]. In this study, we searched for upstream miRNAs of EIF4G2 by using starBase. Ultimately, hsa-miR-26a-5p was identified as a potential regulatory miRNA for EIF4G2 in GC. Our study found that hsa-miR-26a-5p was negatively correlated with EIF4G2 and was significantly downregulated in GC, and its low expression predicted unfavorable OS. In addition, patients with advanced cancer have a worse prognosis when hsamiR-26a-5p expression is reduced. Several previous studies have demonstrated that the expression level of hsa-miR-26a-5p was decreased in multiple cancers, including colorectal cancer, bladder cancer, intrahepatic cholangiocarcinoma and hepatocellular carcinoma, and also showed that low expression of hsa-miR-26a-5p was associated with tumor metastasis, leading to poor prognosis[43-47]. Based on the above studies $₫$ we hypothesized that hsa-miR-26a-5p may be the most likely upstream miRNA of EIF4G2, modulating the functions of EIF4G2 in GC.

Next, the potential upstream IncRNAs of the hsa-miR-26a-5p/EIF4G2 axis were further investigated based on the ceRNA mechanism. The results showed that TUG1 was markedly upregulated in GC compared with normal controls, and the high expression level predicted poor prognosis, especially in higher cancer stages and grades. There have been many studies on the effect of TUG1 in tumors. For instance, a study reported that TUG1 was overexpressed in prostate cancer and promoted tumor cell migration, invasion and proliferation by negatively modulating miR-26a expression[48]. Similarly, TUG1 was found to be highly expressed in colorectal cancer and facilitated the progression of cancer[49]. A positive role for TUG1 has also been discovered in pancreatic cancer, esophageal cancer and ovarian cancer[50-52]. In recent years, TUG1 had been reported to act as a carcinogene in GC. Studies have pointed out that TUG1 was significantly overexpressed in GC and accelerated cell metastasis, invasion and proliferation, resulting in poor prognosis[53-56].These results indicate that the TUG1/hsa-miR-26a$5 p / E I F 4 G 2$ axis is a novel regulatory pathway involved GC development.

\section{Conclusion}

In conclusion, we elucidated that EIF4G2 was upregulated in GC and showed that elevated expression of EIF4G2 indicated an unfavorable prognosis. Moreover, our findings showed that EIF4G2 expression may be involved in the modulation of tumor immune cell infiltration. In addition, we also assessed the potential upstream regulatory mechanisms of EIF4G2 in GC and found that the TUG1/hsa-miR-26a-5p/EIF4G2 axis was the most likely regulatory pathway. However, large-scale basic experiments and clinical trials are needed to validate these results. EIF4G2 is positively associated with the occurrence and development of GC and should be considered a prognosis biomarker and potential new therapeutic target.

\section{Abbreviations}

EIF4G2: Eukaryotic translation initiation factor 4 gamma 2; GC: gastric cancer; ROC: Receiver Operator Characteristic; TUG1: Taurine Up-Regulated 1; elFs: eukaryotic translation initiation factors; DLBCL: Diffuse large B-cell lymphoma; TIMER2.0: Tumor Immune Estimation Resource 2.0; GEPIA: Gene Expression Profiling Interactive Analysis; TISIDB: Tumor-Immune System

Page 7/22 
Interaction database; miRNAs: microRNAs; IncRNAs: long non-coding RNAs; TIICs: tumor-infiltrating immune cells; DCs:

dendritic cells; TCGA: The Cancer Genome Atlas; GTEx: Genotype-Tissue Expression; KM plotter: Kaplan-Meier plotter; GEO: Gene Expression Omnibus; RNA-Seq: RNA sequencing; GO: Gene Ontology; KEGG: Kyoto Encyclopedia of Genes and Genomes; TILs: tumor-infiltrating lymphocytes; TPM: transcripts per million; OS: overall survival; PPS: post progression survival; FP: first progression; AUC: area under the curve; BP: biological process; CC: cellular component; MF: molecular function; TAM: tumorassociated macrophage; ceRNA: competing endogenous RNA; DSS: disease-specific survival; DAP5: death associated protein 5; COPB1: COPI coat complex subunit beta 1

\section{Declarations}

\section{Ethics approval and consent to participate}

Not applicable.

\section{Consent for publication}

Not applicable.

\section{Availability of data and materials}

The datasets used and/or analyzed during the current study are available from the corresponding author on reasonable request. TIMER2.0( http://timer.cistrome.org/ ); GEPIA( http://gepia.cancer-pku.cn/ ); UALCAN( http://ualcan.path.uab.edu/ ); KM plotter(http://kmplot.com/analysis/index.php?p=background) ;LinkedOmics(http://www.linkedomics.org/login.php);TISIDB(http://cis.hku.hk/TISIDB/);StarBase( https://starbase.sysu.edu.cn/ ) .

\section{Competing interests}

The authors declare that they have no competing interests.

\section{Funding}

This work was not supported.

\section{Authors' contributions}

LF and ZW conceived and designed the research. FJ, GW and LS participated in data acquisition and analysis. LF wrote the paper. CG, JW and JZ revised the article. All authors read and approved the final manuscript.

\section{Acknowledgments}

Not applicable.

\section{Authors' information}

${ }^{1}$ Department of Gastroenterology, Putuo People's Hospital, Tongji University, Shanghai 200060, China. ${ }^{2}$ Department of Gastroenterology, Tongji Hospital, Tongji University School of Medicine, Shanghai 200065, China. ${ }^{3}$ Department of Gastroenterology, Shanghai Tenth People's Hospital, Tongji University, Shanghai 200072, China.

\section{References}

1. Bray F, Ferlay J, Soerjomataram I, Siegel RL, Torre LA, Jemal A: Global cancer

2. statistics 2018: GLOBOCAN estimates of incidence and mortality worldwide for 36 cancers in 185 countries. CA Cancer J Clin 2018, 68(6):394-424.https://doi.org/10.3322/caac.21492 
3. Sung H, Ferlay J, Siegel RL, Laversanne M, Soerjomataram I, Jemal A, Bray F: Global Cancer Statistics 2020: GLOBOCAN Estimates of Incidence and Mortality Worldwide for 36 Cancers in 185 Countries. CA Cancer J Clin 2021, 71(3):209249.https://doi.org/10.3322/caac. 21660

4. Thrift AP, El-Serag HB: Burden of Gastric Cancer. Clin Gastroenterol Hepatol 2020, 18(3):534542.https://doi.org/10.1016/j.cgh.2019.07.045

5. Correa P: Gastric cancer: overview. Gastroenterol Clin North Am 2013, 42(2):211217.https://doi.org/10.1016/j.gtc.2013.01.002

6. Allemani C, Weir HK, Carreira H, Harewood R, Spika D, Wang X-S, Bannon F, Ahn JV, Johnson CJ, Bonaventure A et al: Global surveillance of cancer survival 1995-2009: analysis of individual data for 25†676†887 patients from 279 populationbased registries in 67 countries (CONCORD-2). The Lancet 2015, 385(9972):977-1010.https://doi.org/10.1016/s01406736(14)62038-9

7. Golob-SchwarzI N, Krassnig S, Toeglhofer AM, Park YN, Gogg-Kamerer M, Vierlinger K, Schroder F, Rhee H, Schicho R, Fickert $P$ et al: New liver cancer biomarkers: PI3K/AKT/mTOR pathway members and eukaryotic translation initiation factors. Eur $\mathrm{J}$ Cancer 2017, 83:56-70.https://doi.org/10.1016/j.ejca.2017.06.003

8. Gantenbein N, Bernhart E, Anders I, Golob-Schwarzl N, Krassnig S, Wodlej C, Brcic L, Lindenmann J, Fink-Neuboeck N, Gollowitsch F et al: Influence of eukaryotic translation initiation factor 6 on non-small cell lung cancer development and progression. Eur J Cancer 2018, 101:165-180.https://doi.org/10.1016/j.ejca.2018.07.001

9. Skofler C, Kleinegger F, Krassnig S, Birkl-Toeglhofer AM, Singer G, Till H, Benesch M, Cencic R, Porco JA, Jr., Pelletier J et al: Eukaryotic Translation Initiation Factor 4Al: A Potential Novel Target in Neuroblastoma. Cells 2021, 10(2).https://doi.org/10.3390/cells10020301

10. Liberman N, Gandin V, Svitkin YV, David M, Virgili G, Jaramillo M, Holcik M, Nagar B, Kimchi A, Sonenberg N: DAP5 associates with elF2beta and elF4AI to promote Internal Ribosome Entry Site driven translation. Nucleic Acids Res 2015, 43(7):3764-3775.https://doi.org/10.1093/nar/gkv205

11. Lee SH, McCormick F: p97/DAP5 is a ribosome-associated factor that facilitates protein synthesis and cell proliferation by modulating the synthesis of cell cycle proteins. EMBO J 2006, 25(17):40084019.https://doi.org/10.1038/sj.emboj.7601268

12. Lewis SM, Cerquozzi S, Graber TE, Ungureanu NH, Andrews M, Holcik M: The elF4G homolog DAP5/p97 supports the translation of select mRNAs during endoplasmic reticulum stress. Nucleic Acids Res 2008, 36(1):168178.https://doi.org/10.1093/nar/gkm1007

13. Xie X, Li YS, Xiao WF, Deng ZH, He HB, Liu Q, Luo W: MicroRNA-379 inhibits the proliferation, migration and invasion of human osteosarcoma cells by targetting EIF4G2. Biosci Rep 2017, 37(3).https://doi.org/10.1042/BSR20160542

14. Mazan-Mamczarz K, Zhao XF, Dai B, Steinhardt JJ, Peroutka RJ, Berk KL, Landon AL, Sadowska M, Zhang Y, Lehrmann E et al: Down-regulation of elF4GIl by miR-520c-3p represses diffuse large B cell lymphoma development. PLoS Genet 2014, 10(1):e1004105.https://doi.org/10.1371/journal.pgen.1004105

15. Buim ME, Soares FA, Sarkis AS, Nagai MA: The transcripts of SFRP1,CEP63 and EIF4G2 genes are frequently downregulated in transitional cell carcinomas of the bladder. Oncology 2005, 69(6):445454.https://doi.org/10.1159/000090984

16. Li T, Fu J, Zeng Z, Cohen D, Li J, Chen Q, Li B, Liu XS: TIMER2.0 for analysis of tumor-infiltrating immune cells. Nucleic Acids Res 2020, 48(W1):W509-W514.https://doi.org/10.1093/nar/gkaa407

17. Tang Z, Li C, Kang B, Gao G, Li C, Zhang Z: GEPIA: a web server for cancer and normal gene expression profiling and interactive analyses. Nucleic Acids Res 2017, 45(W1):W98-W102.https://doi.org/10.1093/nar/gkx247

18. Chandrashekar DS, Bashel B, Balasubramanya SAH, Creighton CJ, Ponce-Rodriguez I, Chakravarthi B, Varambally S: UALCAN: A Portal for Facilitating Tumor Subgroup Gene Expression and Survival Analyses. Neoplasia 2017, 19(8):649658.https://doi.org/10.1016/j.neo.2017.05.002

19. Gyorffy B, Lanczky A, Eklund AC, Denkert C, Budczies J, Li Q, Szallasi Z: An online survival analysis tool to rapidly assess the effect of 22,277 genes on breast cancer prognosis using microarray data of 1,809 patients. Breast Cancer Res Treat 
2010, 123(3):725-731.https://doi.org/10.1007/s10549-009-0674-9

20. Vasaikar SV, Straub P, Wang J, Zhang B: LinkedOmics: analyzing multi-omics data within and across 32 cancer types. Nucleic Acids Res 2018, 46(D1):D956-D963.https://doi.org/10.1093/nar/gkx1090

21. Ru B, Wong CN, Tong Y, Zhong JY, Zhong SSW, Wu WC, Chu KC, Wong CY, Lau CY, Chen I et al: TISIDB: an integrated repository portal for tumor-immune system interactions. Bioinformatics 2019, 35(20):42004202.https://doi.org/10.1093/bioinformatics/btz210

22. Li JH, Liu S, Zhou H, Qu LH, Yang JH: starBase v2.0: decoding miRNA-ceRNA, miRNA-ncRNA and protein-RNA interaction networks from large-scale CLIP-Seq data. Nucleic Acids Res 2014, 42(Database issue):D9297.https://doi.org/10.1093/nar/gkt1248

23. Hinnebusch AG, Lorsch JR: The mechanism of eukaryotic translation initiation: new insights and challenges. Cold Spring Harb Perspect Biol 2012, 4(10).https://doi.org/10.1101/cshperspect.a011544

24. Gradi A, Imataka H, Svitkin Y, Rom E, Raught B, Morino S, Sonenberg N: A novel functional human eukaryotic translation initiation factor 4G. Molecular and cellular biology 1998, 18(1):334-342.https://doi.org/10.1128/mcb.18.1.334

25. Emmrich S, Engeland F, El-Khatib M, Henke K, Obulkasim A, Schoning J, Katsman-Kuipers JE, Michel Zwaan C, Pich A, Stary $J$ et al: miR-139-5p controls translation in myeloid leukemia through EIF4G2. Oncogene 2016, 35(14):18221831.https://doi.org/10.1038/onc.2015.247

26. Zhu M, Wang P, Feng F, Li MY: LRF inhibits p53 expression in colon cancer cells via modulating DAP5 activity. Cell Biochem Funct 2017, 35(7):401-406.https://doi.org/10.1002/cbf.3287

27. Chai Y, Xie M: LINC01579 promotes cell proliferation by acting as a ceRNA of miR-139-5p to upregulate EIF4G2 expression in glioblastoma. J Cell Physiol 2019, 234(12):23658-23666.https://doi.org/10.1002/jcp.28933

28. Li S, Shao J, Lou G, Wu C, Liu Y, Zheng M: MiR-144-3p-mediated dysregulation of EIF4G2 contributes to the development of hepatocellular carcinoma through the ERK pathway. J Exp Clin Cancer Res 2021, 40(1):53.https://doi.org/10.1186/s13046021-01853-6

29. Chen H, Li K, Li Y, Xie P, He J, Zhang H: An integrative pan-cancer analysis of COPB1 based on data mining. Cancer Biomark 2021, 30(1):13-27.https://doi.org/10.3233/CBM-200398

30. Zhang B, Yin Y, Hu Y, Zhang J, Bian Z, Song M, Hua D, Huang Z: MicroRNA-204-5p inhibits gastric cancer cell proliferation by downregulating USP47 and RAB22A. Med Oncol 2015, 32(1):331.https://doi.org/10.1007/s12032-014-0331-y

31. Jin Z, Jiang W, Jiao F, Guo Z, Hu H, Wang L, Wang L: Decreased expression of histone deacetylase 10 predicts poor prognosis of gastric cancer patients. International journal of clinical and experimental pathology 2014, 7(9):5872-5879

32. Zhang J, Yan Y, Yang Y, Wang L, Li M, Wang J, Liu X, Duan X, Wang J: High Infiltration of Tumor-Associated Macrophages Influences Poor Prognosis in Human Gastric Cancer Patients, Associates With the Phenomenon of EMT. Medicine (Baltimore) 2016, 95(6):e2636.https://doi.org/10.1097/MD.0000000000002636

33. Pollard J: Tumour-educated macrophages promote tumour progression and metastasis. Nature reviews Cancer 2004, 4(1):71-78.https://doi.org/10.1038/nrc1256

34. Zhang W, Gu J, Chen J, Zhang P, Ji R, Qian H, Xu W, Zhang X: Interaction with neutrophils promotes gastric cancer cell migration and invasion by inducing epithelial-mesenchymal transition. Oncol Rep 2017, 38(5):29592966.https://doi.org/10.3892/or.2017.5942

35. Zhang X, Shi H, Yuan X, Jiang P, Qian H, Xu W: Tumor-derived exosomes induce N2 polarization of neutrophils to promote gastric cancer cell migration. Mol Cancer 2018, 17(1):146.https://doi.org/10.1186/s12943-018-0898-6

36. Liu T, Peng L, Yu P, Zhao Y, Shi Y, Mao X, Chen W, Cheng P, Wang T, Chen N et al: Increased circulating Th22 and Th17 cells are associated with tumor progression and patient survival in human gastric cancer. J Clin Immunol 2012, 32(6):13321339.https://doi.org/10.1007/s10875-012-9718-8

37. Liu XS, Lin XK, Mei Y, Ahmad S, Yan CX, Jin HL, Yu H, Chen C, Lin CZ, Yu JR: Regulatory T Cells Promote Overexpression of Lgr5 on Gastric Cancer Cells via TGF-beta1 and Confer Poor Prognosis in Gastric Cancer. Front Immunol 2019, 10:1741.https://doi.org/10.3389/fimmu.2019.01741

Page $10 / 22$ 
38. Zhang H, Yue R, Zhao P, Yu X, Li J, Ma G, Tang J, Zhang L, Feng L, Sun L et al: Proinflammatory follicular helper T cells promote immunoglobulin $\mathrm{G}$ secretion, suppress regulatory $\mathrm{B}$ cell development, and correlate with worse clinical outcomes in gastric cancer. Tumour biology: the journal of the International Society for Oncodevelopmental Biology and Medicine 2017, 39(6):1010428317705747.https://doi.org/10.1177/1010428317705747

39. Yoon JH, Abdelmohsen K, Gorospe M: Functional interactions among microRNAs and long noncoding RNAs. Semin Cell Dev Biol 2014, 34:9-14.https://doi.org/10.1016/j.semcdb.2014.05.015

40. Ghafouri-Fard S, Shoorei H, Anamag FT, Taheri M: The Role of Non-Coding RNAs in Controlling Cell Cycle Related Proteins in Cancer Cells. Front Oncol 2020, 10:608975.https://doi.org/10.3389/fonc.2020.608975

41. Ji D, Wang Y, Sun B, Yang J, Luo X: Long non-coding RNA MNX1-AS1 promotes hepatocellular carcinoma proliferation and invasion through targeting miR-218-5p/COMMD8 axis. Biochem Biophys Res Commun 2019, 513(3):669674.https://doi.org/10.1016/j.bbrc.2019.04.012

42. Razavi ZS, Tajiknia V, Majidi S, Ghandali M, Mirzaei HR, Rahimian N, Hamblin MR, Mirzaei H: Gynecologic cancers and noncoding RNAs: Epigenetic regulators with emerging roles. Crit Rev Oncol Hematol 2021, 157:103192.https://doi.org/10.1016/j.critrevonc.2020.103192

43. Tay Y, Rinn J, Pandolfi PP: The multilayered complexity of ceRNA crosstalk and competition. Nature 2014, 505(7483):344352.https://doi.org/10.1038/nature12986

44. Vishnubalaji R, Hamam R, Abdulla MH, Mohammed MA, Kassem M, Al-Obeed O, Aldahmash A, Alajez NM: Genome-wide mRNA and miRNA expression profiling reveal multiple regulatory networks in colorectal cancer. Cell Death Dis 2015, 6:e1614.https://doi.org/10.1038/cddis.2014.556

45. Canturk KM, Ozdemir M, Can C, Oner S, Emre R, Aslan H, Cilingir O, Ciftci E, Celayir FM, Aldemir O et al: Investigation of key miRNAs and target genes in bladder cancer using miRNA profiling and bioinformatic tools. Mol Biol Rep 2014, 41(12):8127-8135.https://doi.org/10.1007/s11033-014-3713-5

46. Qin X, Song Y: Bioinformatics Analysis Identifies the Estrogen Receptor 1 (ESR1) Gene and hsa-miR-26a-5p as Potential Prognostic Biomarkers in Patients with Intrahepatic Cholangiocarcinoma. Med Sci Monit 2020, 26:e921815.https://doi.org/10.12659/MSM.921815

47. Liang L, Zeng JH, Wang JY, He RQ, Ma J, Chen G, Cai XY, Hu XH: Down-regulation of miR-26a-5p in hepatocellular carcinoma: A qRT-PCR and bioinformatics study. Pathol Res Pract 2017, 213(12):14941509.https://doi.org/10.1016/j.prp.2017.10.001

48. Chang L, Li K, Guo T: miR-26a-5p suppresses tumor metastasis by regulating EMT and is associated with prognosis in HCC. Clin Transl Oncol 2017, 19(6):695-703.https://doi.org/10.1007/s12094-016-1582-1

49. Yang B, Tang X, Wang Z, Sun D, Wei X, Ding Y: TUG1 promotes prostate cancer progression by acting as a ceRNA of miR26a. Biosci Rep 2018, 38(5).https://doi.org/10.1042/BSR20180677

50. Liu Q, Zhang W, Luo L, Han K, Liu R, Wei S, Guo X: Long noncoding RNA TUG1 regulates the progression of colorectal cancer through miR-542-3p/TRIB2 axis and Wnt/beta-catenin pathway. Diagn Pathol 2021, 16(1):47.https://doi.org/10.1186/s13000-021-01101-7

51. Xu K, Zhang L: Inhibition of TUG1/miRNA-299-3p Axis Represses Pancreatic Cancer Malignant Progression via Suppression of the Notch1 Pathway. Dig Dis Sci 2020, 65(6):1748-1760.https://doi.org/10.1007/s10620-019-05911-0

52. Zong M, Feng W, Wan L, Yu X, Yu W: LncRNA TUG1 promotes esophageal cancer development through regulating PLK1 expression by sponging miR-1294. Biotechnol Lett 2020, 42(12):2537-2549.https://doi.org/10.1007/s10529-020-02984-0

53. Zhan FL, Chen CF, Yao MZ: LncRNA TUG1 facilitates proliferation, invasion and stemness of ovarian cancer cell via miR186-5p/ZEB1 axis. Cell Biochem Funct 2020, 38(8):1069-1078.https://doi.org/10.1002/cbf.3544

54. Ren K, Li Z, Li Y, Zhang W, Han X: Long Noncoding RNA Taurine-Upregulated Gene 1 Promotes Cell Proliferation and Invasion in Gastric Cancer via Negatively Modulating miRNA-145-5p. Oncol Res 2017, 25(5):789798.https://doi.org/10.3727/096504016X14783677992682

55. Zhang E, He X, Yin D, Han L, Qiu M, Xu T, Xia R, Xu L, Yin R, De W: Increased expression of long noncoding RNA TUG1 predicts a poor prognosis of gastric cancer and regulates cell proliferation by epigenetically silencing of p57. Cell Death Dis 
2016, 7:e2109.https://doi.org/10.1038/cddis.2015.356

56. Ji TT, Huang X, Jin J, Pan SH, Zhuge XJ: Inhibition of long non-coding RNA TUG1 on gastric cancer cell transference and invasion through regulating and controlling the expression of miR-144/c-Met axis. Asian Pac J Trop Med 2016, 9(5):508512.https://doi.org/10.1016/j.apjtm.2016.03.026

57. Baratieh Z, Khalaj Z, Honardoost M, Emadi-Baygi M, Khanahmad H, Salehi M, Nikpour P: Aberrant expression of PIncRNA-1 and TUG1: potential biomarkers for gastric cancer diagnosis and clinically monitoring cancer progression. Biomarkers in medicine 2017, 11(12):1077-1090.https://doi.org/10.2217/bmm-2017-0090

\section{Tables}

Table1 Correlation analysis between EIF4G2 and markers of immune cells in GC 


\begin{tabular}{|c|c|c|c|c|c|}
\hline \multirow[t]{2}{*}{ Description } & \multirow[t]{2}{*}{ Gene marker } & \multicolumn{2}{|l|}{ None } & \multicolumn{2}{|l|}{ Purity } \\
\hline & & Cor & $P$ & Partial. cor & Partial. $P$ \\
\hline \multirow[t]{2}{*}{$B$ cell } & CD19 & 0.019 & 7.04E-01 & 0.015 & 7.75E-01 \\
\hline & CD38 & 0.196 & $6.20 \mathrm{E}-05$ & 0.201 & $8.35 \mathrm{E}-05^{\star \star \star}$ \\
\hline \multirow[t]{2}{*}{ CD8 T Cell } & CD8A & 0.081 & 9.97E-02 & 0.088 & 8.57E-02 \\
\hline & CD8B & 0.03 & 5.43E-01 & 0.046 & 3.69E-01 \\
\hline CD4 T Cell & CD4 & 0.169 & $5.34 \mathrm{E}-04^{\star \star \star}$ & 0.179 & $4.64 \mathrm{E}-04^{\star \star \star}$ \\
\hline \multirow[t]{3}{*}{ M1 Macrophage } & NOS2 & 0.086 & $8.06 \mathrm{E}-02$ & 0.105 & $4.17 \mathrm{E}-02^{*}$ \\
\hline & IRF5 & 0.116 & $1.86 \mathrm{E}-02^{*}$ & 0.106 & $3.99 \mathrm{E}-02^{*}$ \\
\hline & PTGS2 & 0.229 & $2.60 \mathrm{E}-06^{\star \star \star}$ & 0.242 & $1.91 \mathrm{E}-06^{* \star \star}$ \\
\hline \multirow[t]{5}{*}{ M2 Macrophage } & CD163 & 0.34 & $1.37 \mathrm{E}-12^{\star \star \star}$ & 0.347 & $3.64 \mathrm{E}-12^{\star * \star}$ \\
\hline & VSIG4 & 0.169 & $5.63 \mathrm{E}-04^{\star \star \star}$ & 0.178 & $5.10 \mathrm{E}-04^{\star \star \star}$ \\
\hline & MS4A4A & 0.213 & $1.28 \mathrm{E}-05^{\star \star \star}$ & 0.223 & 1.17E-0 $05^{\star \star \star}$ \\
\hline & ARG1 & 0.139 & 4.63E-03** & 0.146 & $4.28 \mathrm{E}-03^{\star *}$ \\
\hline & MRC1 & 0.319 & $3.51 \mathrm{E}-11^{\star \star \star}$ & 0.321 & $1.65 \mathrm{E}-10^{\star \star \star}$ \\
\hline \multirow[t]{4}{*}{ Neutrophil } & CEACAM8 & 0.14 & $4.16 \mathrm{E}-03^{\star \star}$ & 0.155 & $2.54 \mathrm{E}-03^{\star \star}$ \\
\hline & ITGAM & 0.251 & $2.41 \mathrm{E}-07^{\star \star \star}$ & 0.252 & $6.63 \mathrm{E}-07^{\star \star \star}$ \\
\hline & CCR7 & 0.104 & $3.48 \mathrm{E}-02^{*}$ & 0.12 & $1.91 \mathrm{E}-02^{*}$ \\
\hline & MPO & 0.191 & $9.35 \mathrm{E}-05^{\star \star \star}$ & 0.225 & $9.71 \mathrm{E}-06^{\star \star \star}$ \\
\hline \multirow[t]{5}{*}{ DC } & HLA-DRA & 0.052 & $2.90 \mathrm{E}-01$ & 0.057 & 2.67E-01 \\
\hline & HLA-DPA1 & 0.034 & 4.89E-01 & 0.034 & $5.05 \mathrm{E}-01$ \\
\hline & NRP1 & 0.34 & $1.40 \mathrm{E}-12^{\star \star \star}$ & 0.342 & $7.89 \mathrm{E}-12^{\star \star \star}$ \\
\hline & ITGAX & 0.232 & $2.00 \mathrm{E}-06^{\star \star \star}$ & 0.247 & $1.09 \mathrm{E}-06^{\star \star \star}$ \\
\hline & CD141 & 0.169 & $5.34 \mathrm{E}-04^{\star \star \star}$ & 0.165 & $1.23 \mathrm{E}-03^{\star \star}$ \\
\hline \multirow[t]{2}{*}{ Monocyte } & CSF1R & 0.23 & $2.43 \mathrm{E}-06^{\star \star \star}$ & 0.231 & $5.47 \mathrm{E}-06^{\star \star \star}$ \\
\hline & CD86 & 0.194 & $6.68 \mathrm{E}-05^{\star \star \star}$ & 0.214 & $2.69 \mathrm{E}-05^{\star \star \star}$ \\
\hline \multirow[t]{6}{*}{ NK cell } & KIR2DS4 & 0.114 & $2.06 \mathrm{E}-02^{*}$ & 0.12 & $1.96 \mathrm{E}-02^{*}$ \\
\hline & KIR3DL3 & 0.032 & 5.11E-01 & 0.045 & $3.86 \mathrm{E}-01$ \\
\hline & KIR3DL2 & 0.114 & $2.04 \mathrm{E}-02^{*}$ & 0.132 & $1.03 \mathrm{E}-02^{*}$ \\
\hline & KIR3DL1 & 0.147 & $2.74 \mathrm{E}-03^{* *}$ & 0.151 & $3.24 \mathrm{E}-03^{* *}$ \\
\hline & KIR2DL4 & 0.102 & $3.75 \mathrm{E}-02^{*}$ & 0.112 & $2.91 \mathrm{E}-02^{*}$ \\
\hline & KIR2DL3 & 0.163 & $8.89 \mathrm{E}-04^{\star \star \star}$ & 0.173 & $7.43 \mathrm{E}-04^{\star \star \star}$ \\
\hline
\end{tabular}

Page 13/22 


\begin{tabular}{|c|c|c|c|c|c|}
\hline & KIR2DL1 & 0.164 & $7.83 \mathrm{E}-04^{\star \star \star}$ & 0.189 & $2.12 \mathrm{E}-04^{\star \star \star}$ \\
\hline \multirow[t]{2}{*}{ T cell } & CD3D & 0.022 & $6.51 \mathrm{E}-01$ & 0.035 & $5.00 \mathrm{E}-01$ \\
\hline & $\mathrm{CD} 2$ & 0.128 & $9.19 \mathrm{E}-03^{\star *}$ & 0.145 & $4.73 \mathrm{E}-03^{\star \star}$ \\
\hline \multirow[t]{5}{*}{ T cell exhaustion } & CTLA4 & 0.209 & $1.91 \mathrm{E}-05^{\star \star \star}$ & 0.229 & $6.48 \mathrm{E}-06^{\star \star \star}$ \\
\hline & LAG3 & 0.063 & $2.02 \mathrm{E}-01$ & 0.061 & $2.38 \mathrm{E}-01$ \\
\hline & HAVCR2 & 0.211 & $1.53 \mathrm{E}-05^{\star \star \star}$ & 0.22 & $1.62 \mathrm{E}-05^{\star \star \star}$ \\
\hline & GZMB & 0.057 & $2.43 E-01$ & 0.057 & $2.66 \mathrm{E}-01$ \\
\hline & PDCD1 & 0.076 & $1.22 \mathrm{E}-01$ & 0.093 & 7.16E-02 \\
\hline \multirow[t]{2}{*}{ TAM } & IL10 & 0.234 & $1.50 \mathrm{E}-06^{\star \star \star}$ & 0.246 & $1.24 \mathrm{E}-06^{\star \star \star}$ \\
\hline & $\mathrm{CD} 68$ & 0.109 & $2.67 \mathrm{E}-02^{*}$ & 0.1 & $5.11 \mathrm{E}-02$ \\
\hline \multirow[t]{2}{*}{ Tfh } & BCL6 & 0.238 & $9.36 \mathrm{E}-07^{\star \star \star}$ & 0.237 & $3.01 \mathrm{E}-06^{\star \star \star}$ \\
\hline & IL21 & 0.151 & $2.00 \mathrm{E}-03^{\star \star}$ & 0.155 & $2.46 \mathrm{E}-03^{\star \star}$ \\
\hline \multirow[t]{4}{*}{ Th1 } & TBX21 & 0.097 & $4.83 \mathrm{E}-02^{*}$ & 0.114 & $2.71 \mathrm{E}-02^{*}$ \\
\hline & STAT4 & 0.249 & $3.14 \mathrm{E}-07^{\star \star \star}$ & 0.274 & $6.20 \mathrm{E}-08^{\star \star \star}$ \\
\hline & IFNG & 0.163 & $8.73 \mathrm{E}-04^{\star \star \star}$ & 0.172 & $7.76 \mathrm{E}-04^{\star \star \star}$ \\
\hline & IL13 & 0.017 & $7.29 \mathrm{E}-01$ & 0.024 & $6.48 \mathrm{E}-01$ \\
\hline \multirow[t]{3}{*}{ Th2 } & GATA3 & -0.002 & $9.74 \mathrm{E}-01$ & 0.024 & $6.48 \mathrm{E}-01$ \\
\hline & STAT6 & 0.356 & $7.27 \mathrm{E}-14^{\star \star \star}$ & 0.353 & $1.35 \mathrm{E}-12^{\star \star \star}$ \\
\hline & STAT5A & 0.321 & $2.10 \mathrm{E}-11^{\star \star \star}$ & 0.329 & $5.00 \mathrm{E}-11^{\star \star \star}$ \\
\hline \multirow[t]{2}{*}{ Th17 } & STAT3 & 0.52 & $0.00 \mathrm{E}+00^{\star \star \star}$ & 0.517 & $2.68 \mathrm{E}-27^{\star \star \star}$ \\
\hline & IL17A & 0.085 & $8.50 \mathrm{E}-02$ & 0.084 & $1.03 \mathrm{E}-01$ \\
\hline \multirow[t]{4}{*}{ Treg } & FOXP3 & 0.172 & $4.56 \mathrm{E}-04^{\star \star \star}$ & 0.185 & $2.85 \mathrm{E}-04^{\star \star \star}$ \\
\hline & CCR8 & 0.268 & $2.93 \mathrm{E}-08^{\star \star \star}$ & 0.284 & $1.73 \mathrm{E}-08^{\star \star \star}$ \\
\hline & STAT5B & 0.447 & $0.00 \mathrm{E}+00^{\star \star \star}$ & 0.442 & $1.46 \mathrm{E}-19^{\star \star \star}$ \\
\hline & TGFB1 & 0.115 & $1.93 \mathrm{E}-02^{*}$ & 0.113 & $2.76 \mathrm{E}-02^{\star}$ \\
\hline
\end{tabular}

Cor, R value of Spearman's correlation; None, correlation without adjustment. Purity, correlation adjusted by purity. ${ }^{*} \mathrm{P}<0.05$, ${ }^{\star \star P}$ $<0.01, \star \star \star P P<0.001$.

Table2 The predicted 15 miRNAs negatively correlated with EIF4G2 expression 


\begin{tabular}{llll} 
Gene & miRNA & R-value & P-value \\
\hline EIF4G2 & hsa-let-7a-5p & -0.117 & $2.43 \mathrm{E}-02^{*}$ \\
\hline EIF4G2 & hsa-let-7f-5p & -0.225 & $1.22 \mathrm{E}-05^{* \star}$ \\
\hline EIF4G2 & hsa-miR-26a-5p & -0.266 & $2.00 \mathrm{E}-07^{\star *}$ \\
\hline EIF4G2 & hsa-miR-26b-5p & -0.137 & $8.35 \mathrm{E}-03^{* *}$ \\
\hline EIF4G2 & hsa-miR-32-5p & -0.113 & $2.95 \mathrm{E}-02^{*}$ \\
\hline EIF4G2 & hsa-miR-101-3p & -0.118 & $2.32 \mathrm{E}-02^{*}$ \\
\hline EIF4G2 & hsa-let-7g-5p & -0.156 & $2.60 \mathrm{E}-03^{* *}$ \\
\hline EIF4G2 & hsa-miR-30b-5p & -0.12 & $2.10 \mathrm{E}-02^{*}$ \\
\hline EIF4G2 & hsa-miR-106b-5p & -0.102 & $4.99 \mathrm{E}-02^{*}$ \\
\hline EIF4G2 & hsa-miR-374a-5p & -0.116 & $2.49 \mathrm{E}-02^{*}$ \\
\hline EIF4G2 & hsa-miR-493-3p & -0.115 & $2.69 \mathrm{E}-02^{*}$ \\
\hline EIF4G2 & hsa-miR-411-5p & -0.138 & $7.67 \mathrm{E}-03^{* *}$ \\
\hline EIF4G2 & hsa-miR-758-3p & -0.119 & $2.20 \mathrm{E}-02^{*}$ \\
\hline EIF4G2 & hsa-miR-340-5p & -0.164 & $1.47 \mathrm{E}-03^{* *}$ \\
\hline EIF4G2 & hsa-miR-374b-5p & -0.181 & $4.43 \mathrm{E}-04^{* *}$
\end{tabular}

${ }^{\star} p<0.05,{ }^{\star \star} p<0.01,{ }^{\star \star \star} p<0.001$.

Table3 The predicted nine IncRNAs negatively correlated with hsa-miR-26a-5p

\begin{tabular}{|c|c|c|c|}
\hline miRNA & IncRNA & $R$ value & $P$ value \\
\hline hsa-miR-26a-5p & NORAD & -0.2 & $1.04 \mathrm{E}-04^{\star \star \star}$ \\
\hline hsa-miR-26a-5p & AC068768.1 & -0.156 & $2.56 \mathrm{E}-03^{\star \star}$ \\
\hline hsa-miR-26a-5p & TUG1 & -0.186 & $3.03 \mathrm{E}-04^{\star \star \star}$ \\
\hline hsa-miR-26a-5p & AC005261.1 & -0.138 & $7.61 \mathrm{E}-03^{\star \star}$ \\
\hline hsa-miR-26a-5p & OIP5-AS1 & -0.146 & $4.64 \mathrm{E}-03^{\star \star}$ \\
\hline hsa-miR-26a-5p & AC000120.1 & -0.124 & $1.71 \mathrm{E}-02^{*}$ \\
\hline hsa-miR-26a-5p & AL139407.1 & -0.194 & $1.72 \mathrm{E}-04^{\star \star \star}$ \\
\hline hsa-miR-26a-5p & AC093297.2 & -0.169 & $1.10 \mathrm{E}-03^{\star \star}$ \\
\hline hsa-miR-26a-5p & EBLN3P & -0.172 & $8.82 \mathrm{E}-04^{\star * *}$ \\
\hline
\end{tabular}

Table4 The predicted nine IncRNAs positively correlated with EIF4G2 


\begin{tabular}{llll} 
IncRNA & mRNA & R value & P value \\
\hline NORAD & EIF4G2 & 0.178 & $5.35 \mathrm{E}-04^{\star \star \star}$ \\
\hline AC068768.1 & EIF4G2 & 0.237 & $3.46 \mathrm{E}-06^{\star \star \star}$ \\
\hline TUG1 & EIF4G2 & 0.396 & $1.46 \mathrm{E}-15^{\star \star \star}$ \\
\hline AC005261.1 & EIF4G2 & 0.133 & $9.78 \mathrm{E}-03^{\star \star}$ \\
\hline OIP5-AS1 & EIF4G2 & 0.19 & $2.14 \mathrm{E}-04^{\star \star *}$ \\
\hline AC000120.1 & EIF4G2 & 0.178 & $5.27 \mathrm{E}-04^{\star \star \star}$ \\
\hline AL139407.1 & EIF4G2 & 0.214 & $3.02 \mathrm{E}-05^{\star \star *}$ \\
\hline AC093297.2 & EIF4G2 & 0.129 & $1.26 \mathrm{E}-02^{\star}$ \\
\hline EBLN3P & EIF4G2 & 0.33 & $5.98 \mathrm{E}-11^{\star \star *}$
\end{tabular}

${ }^{*} p<0.05,{ }^{* *} p<0.01,{ }^{* \star *} p<0.001$.

\section{Figures}




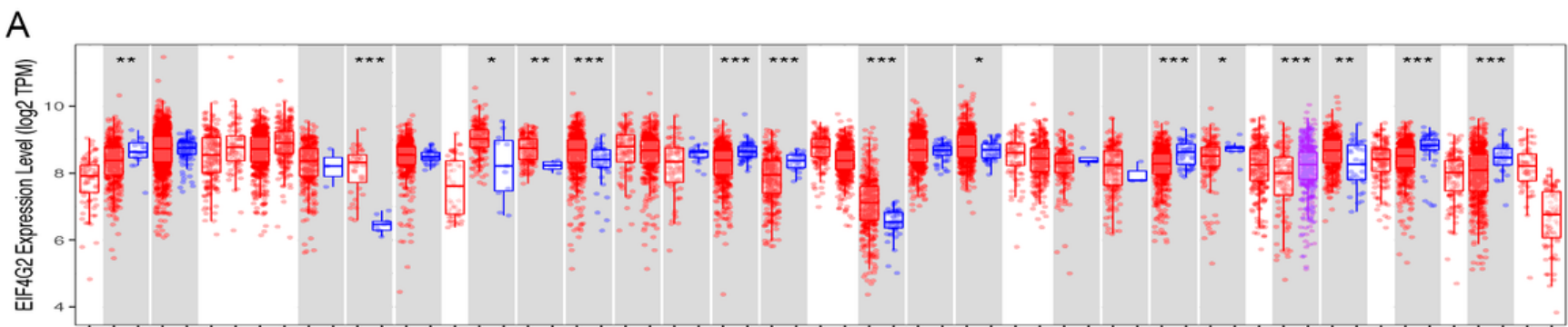

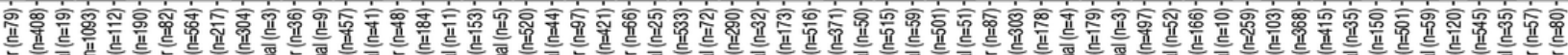

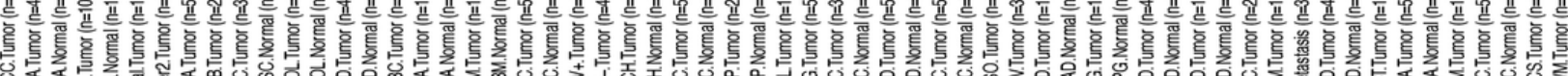

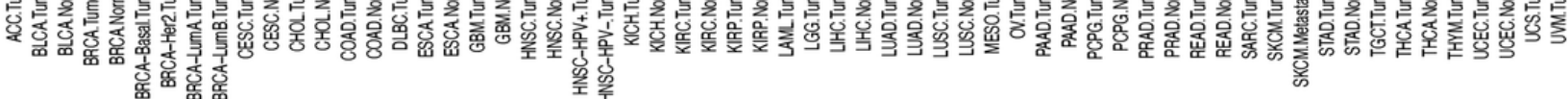

B

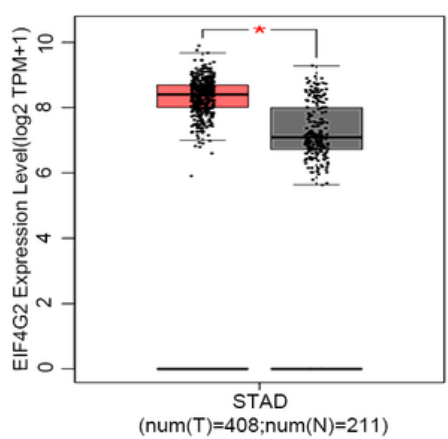

E

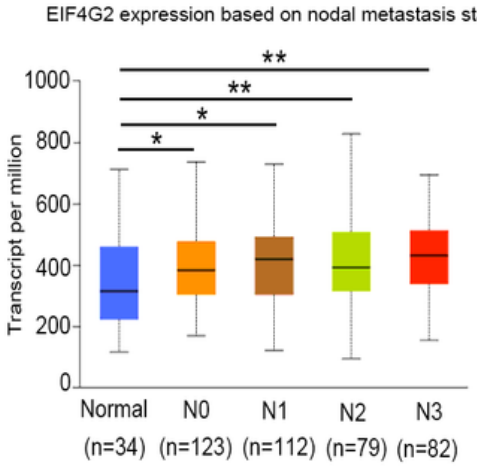

C

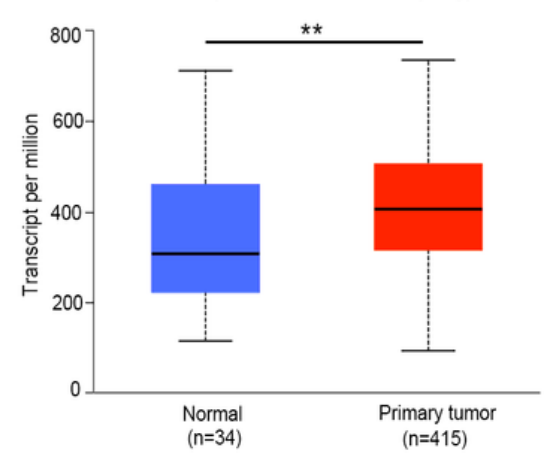

$\mathrm{F}$

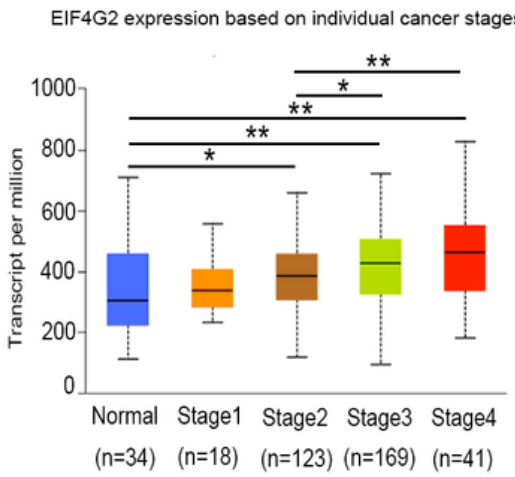

D

EIF4G2 expression based on patient's gender

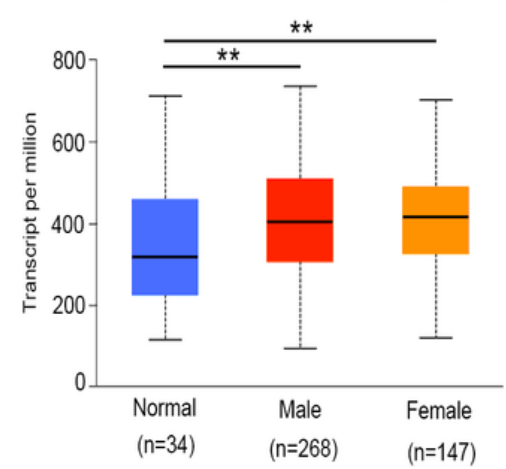

G

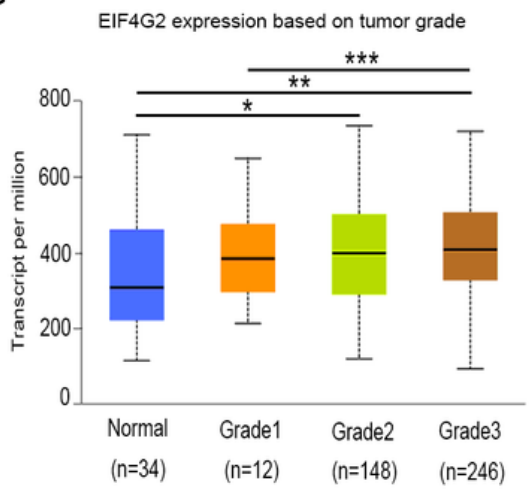

\section{Figure 1}

Expression analysis of EIF4G2 in GC using different online tools. (A) The expression of EIF4G2 in different types of tumor and normal tissues in the TIMER2.0 database. ${ }^{*} P<0.05,{ }^{*} P<0.01,{ }^{*} * P<0.001$. (B) EIF4G2 expression in TCGA GC tissues compared with corresponding TCGA and GTEx normal tissues (GEPIA). ${ }^{*}>0.01$. EIF4G2 expression level in GC (UALCAN) based on sample type (C), patient's gender (D), nodal metastasis status (E), individual cancer stage (F) and tumor grade (G). N0: No regional lymph node metastasis; N1: Metastases in one to three axillary lymph nodes; N2: Metastases in four to nine axillary lymph nodes; N3: Metastases in ten or more axillary lymph nodes. Grade 1: Well differentiated (low grade); Grade 2: Moderately differentiated (intermediate grade); Grade 3: Poorly differentiated (high grade); Grade 4: Undifferentiated (high grade). ${ }^{\star} \mathrm{P}<0.05$, $\star \star P P 0.01, * \star * P<0.001$. 

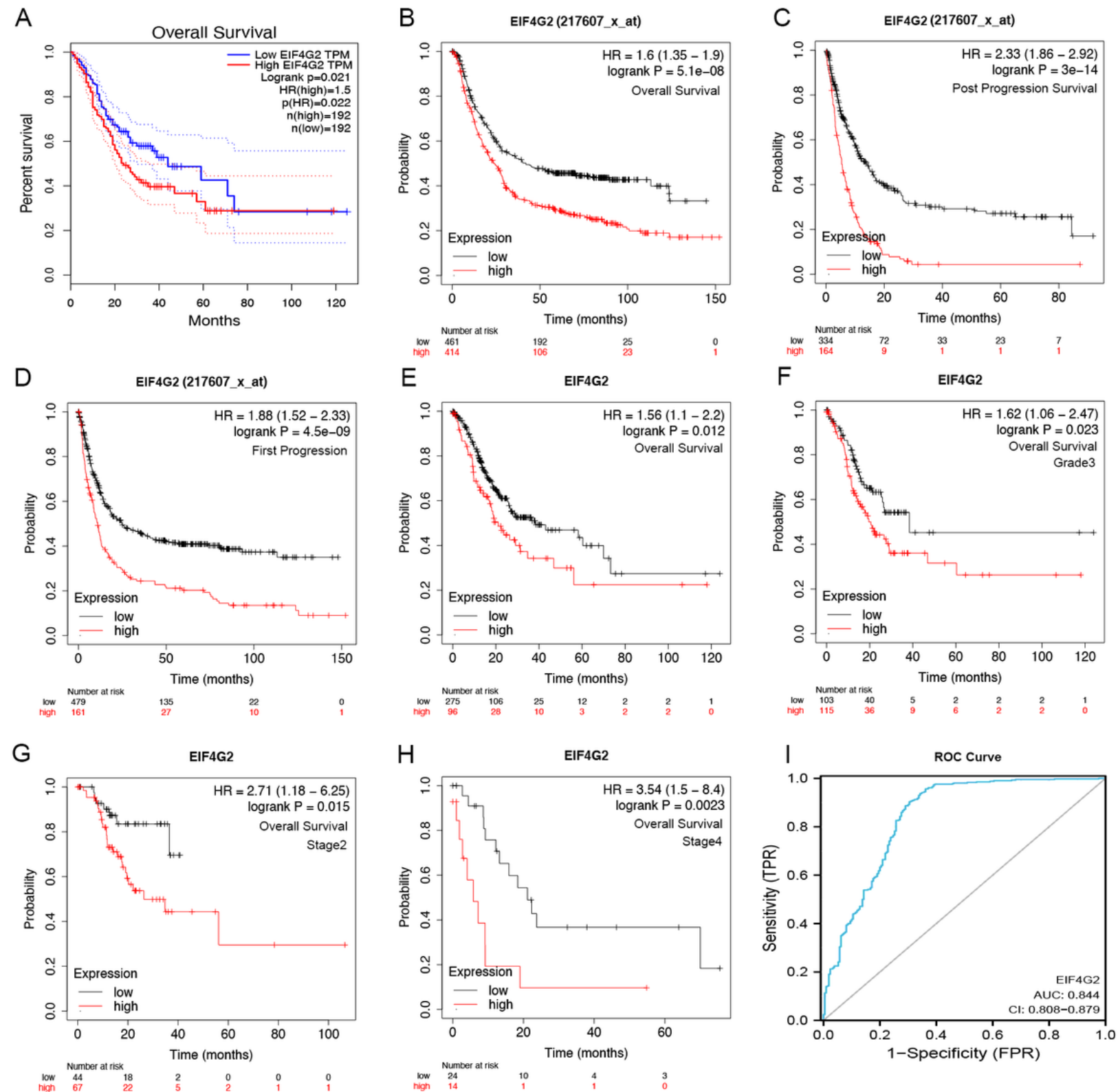

\section{Figure 2}

Prognostic significance and diagnostic value of EIF4G2 expression in GC. (A) The OS survival curves of GC in the GEPIA database. (B-H) Kaplan-Meier curves comparing high and low expression of EIF4G2 in gastric cancer based on gene chips and RNA-seq data. (B-D) OS, PPS, and FP survival curves based on gene chips. (E-H) Survival curves based on RNA-seq data; expression of EIF4G2 was significant overall (E), in grade $3(F)$, stage $2(G)$ and stage $4(H)$ of gastric cancer patients' OS. (I) The diagnostic value of EIF4G2 in GC (ROC curve). Log-rank P-value $<0.05$ was considered statistically significant. Cl, confidence interval; FPR, false positive rate; TPR, true positive rate. 

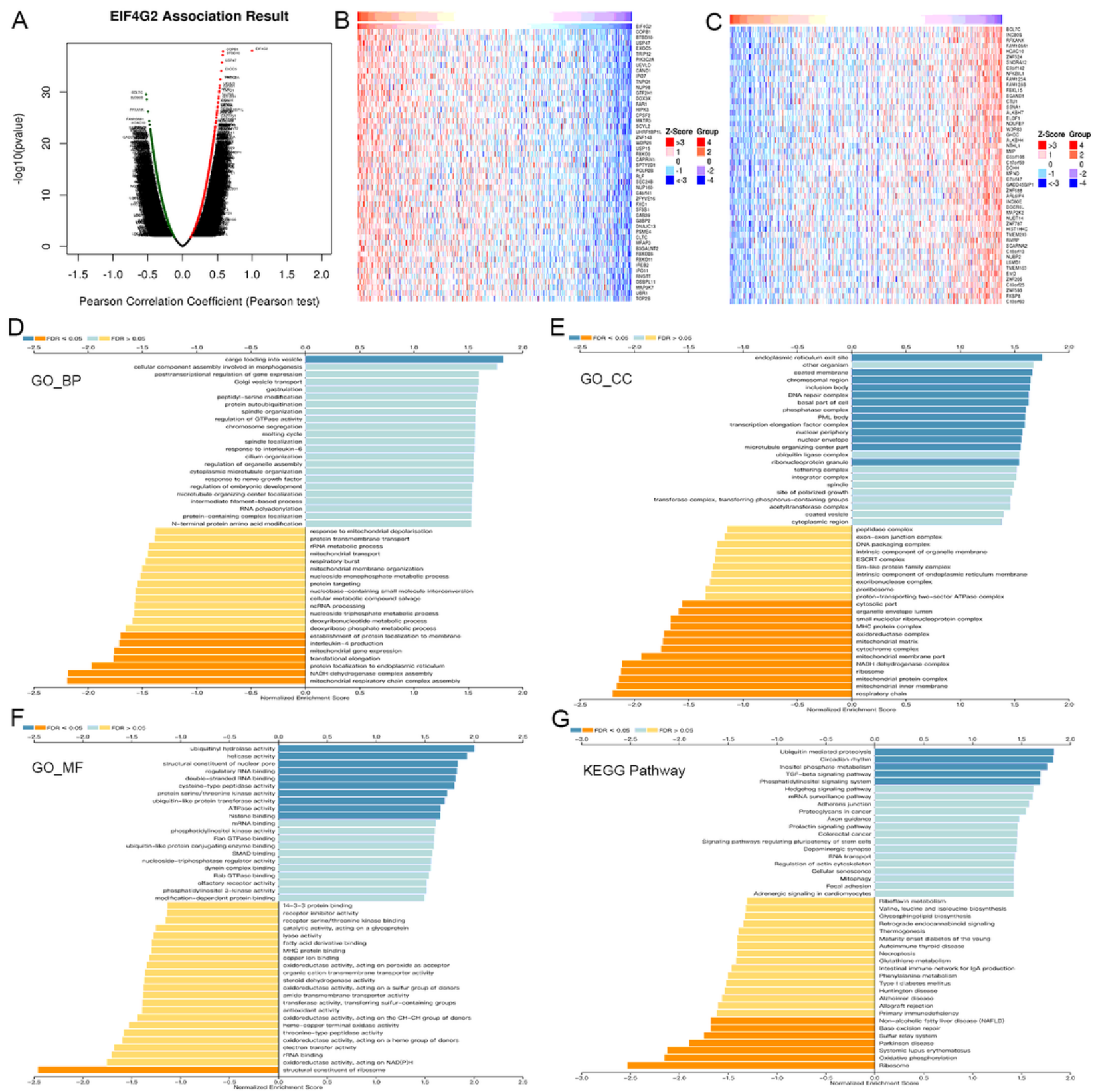

\section{Figure 3}

The co-expressed genes with EIF4G2 in GC from the LinkedOmics database. (A) All significantly associated genes with EIF4G2 distinguished by Pearson test in the GC cohort. (B-C) Heat maps showing the top 50 genes positively and negatively related to EIF4G2 in GC. Red represents positively linked genes and blue represents negatively linked genes. (D-G) GO analysis and KEGG pathways of the genes co-expressed with EIF4G2 in GC showing biological process (D), cellular component (E), molecular function (F) and KEGG pathways (G). 

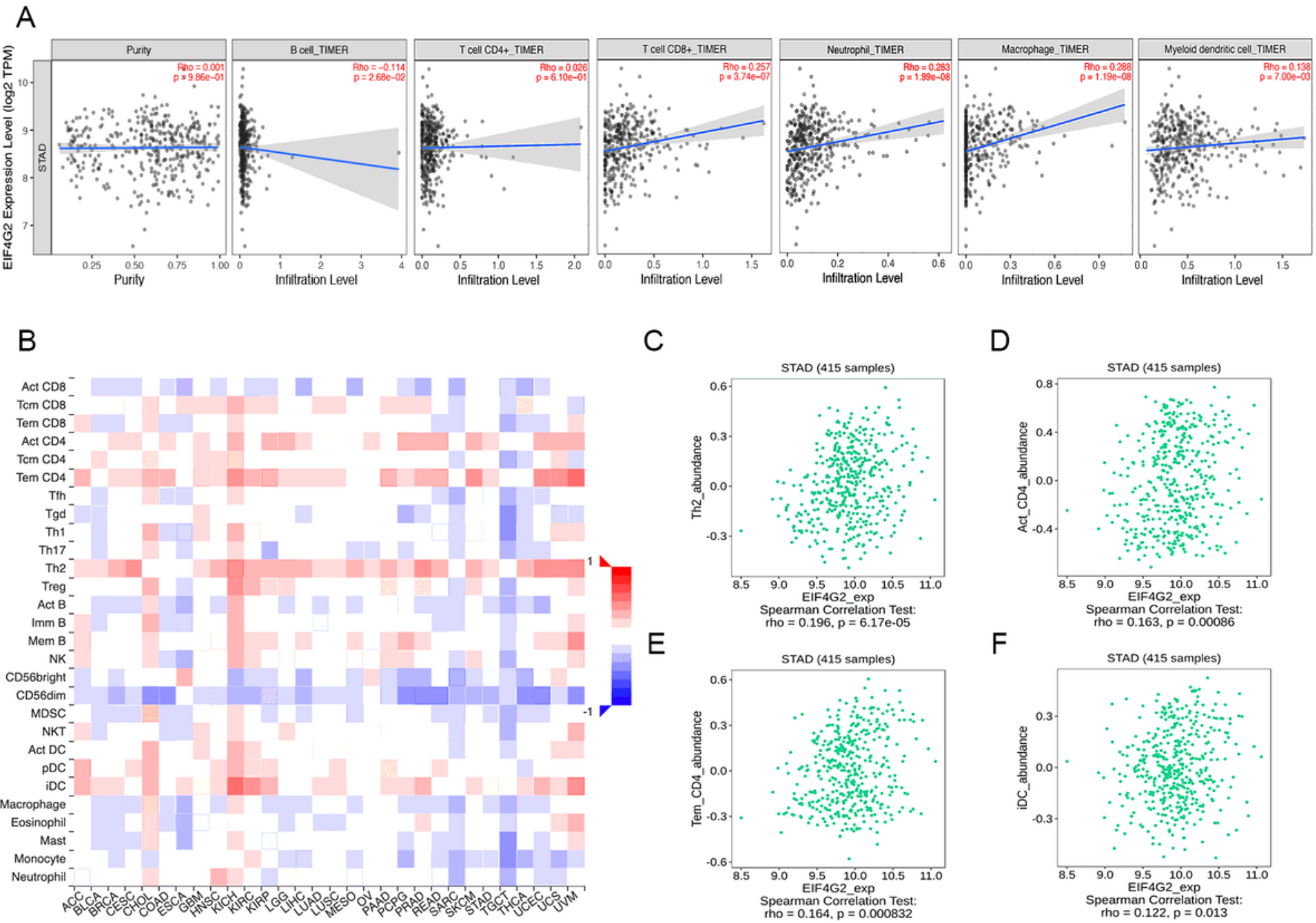

\section{Figure 4}

Correlations of EIF4G2 expression with immune infiltration level. (A) Correlations between expression of EIF4G2 with tumor purity, and infiltration level of B cells, CD4+ T cells, CD8+ T cells, neutrophils, macrophages and DCs in GC (TIMER2.0). (B) Relationship between the expression of EIF4G2 and 28 types of TILs across human cancers from TISIDB. (C-F) Top four TILs displaying the greatest Spearman correlation with EIF4G2 expression in GC. P 0.05 is statistically significant. 
A

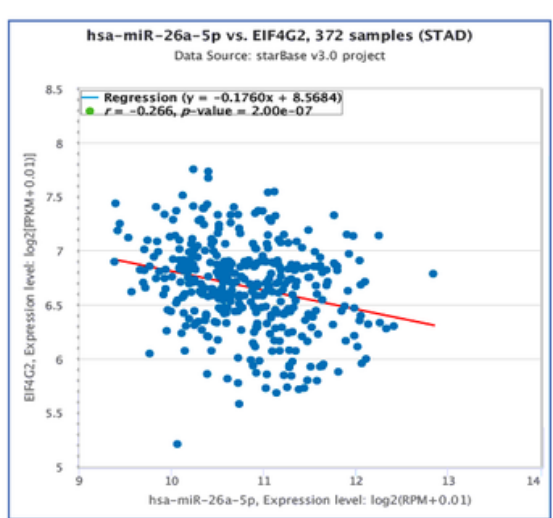

B

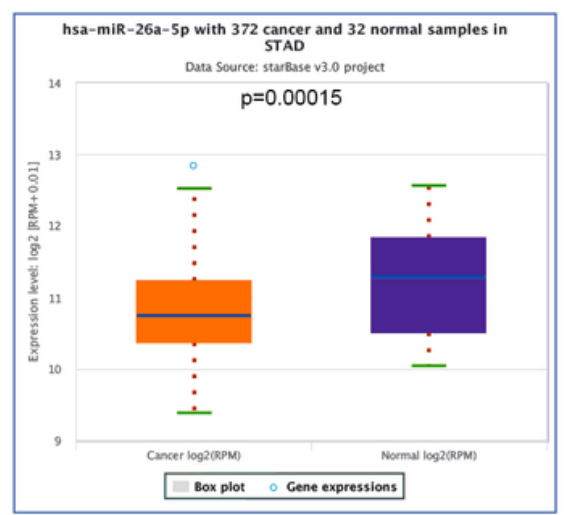

D

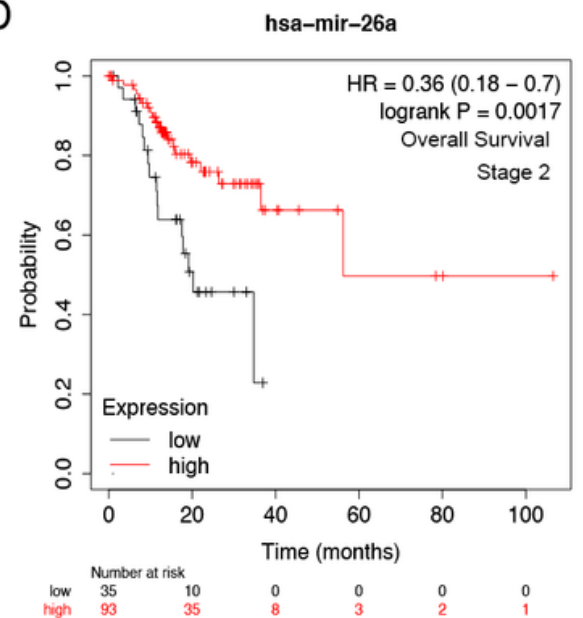

E

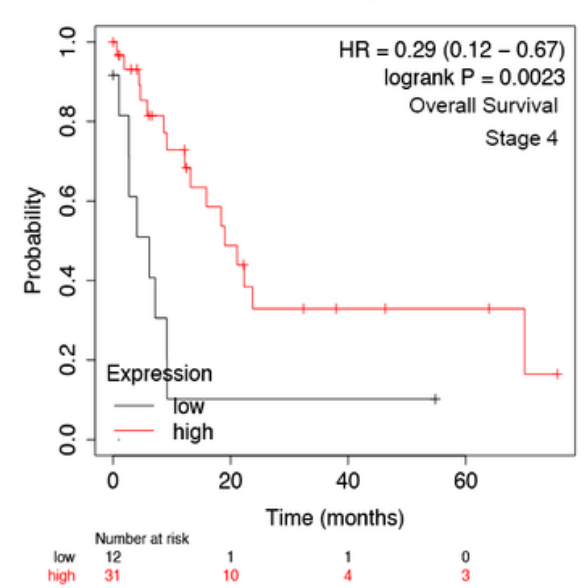

C

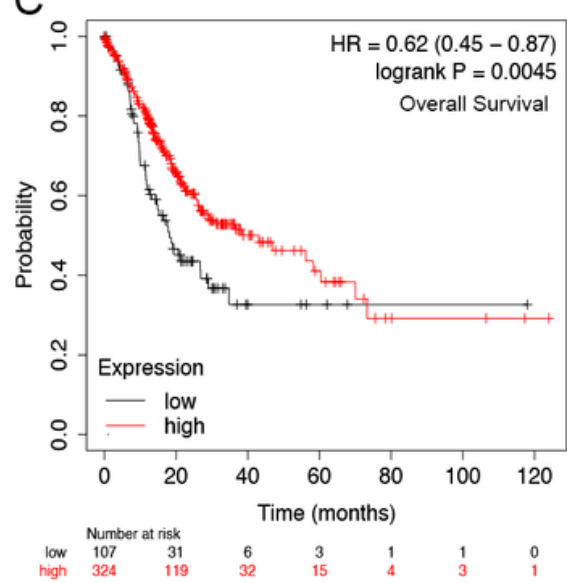

$\mathrm{F}$

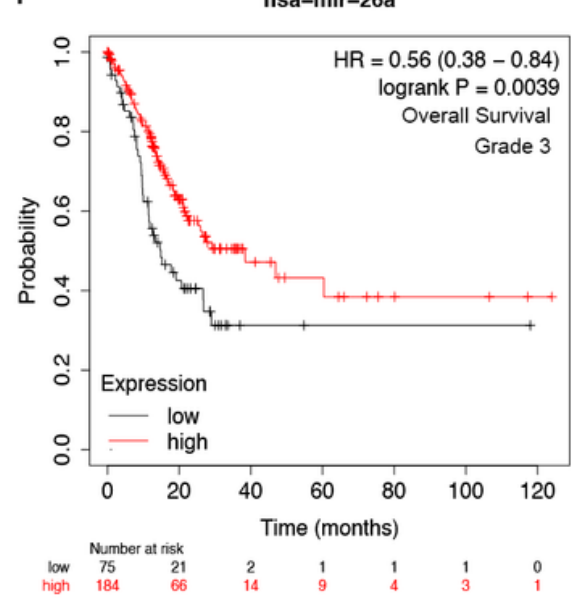

\section{Figure 5}

Identification of hsa-miR-26a-5p as a potential upstream miRNA of EIF4G2 in GC. (A) The expression correlation between hsamiR-26a-5p and EIF4G2 in GC analyzed by the starBase database. (B) The expression of hsa-miR-26a-5p in GC and control normal samples determined by starBase. (C) OS survival curves of hsa-miR-26a-5p in GC. (D-F) Prognostic value of hsa-miR$26 a-5 p$ in GC patients with distinct clinicopathological statuses, including stage $2(D)$, stage $4(E)$ and grade $3(F)$. P $<0.05$ is statistically significant. 
A

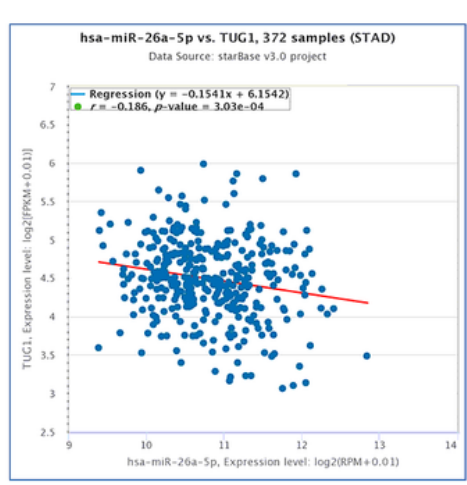

D

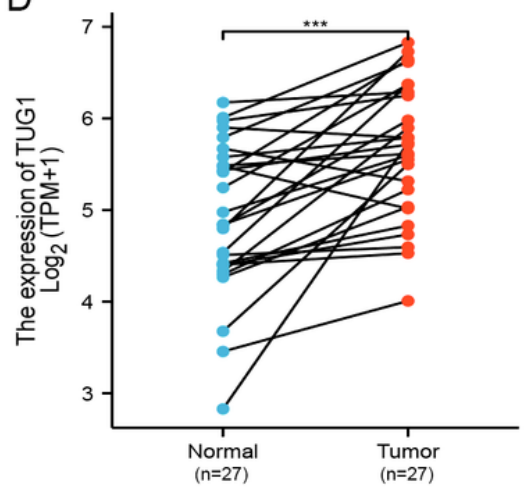

G

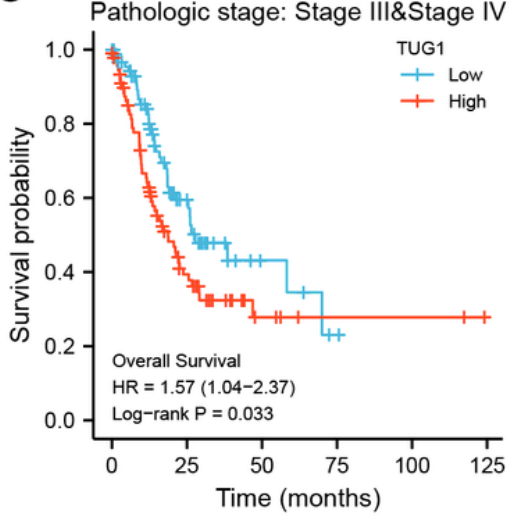

B

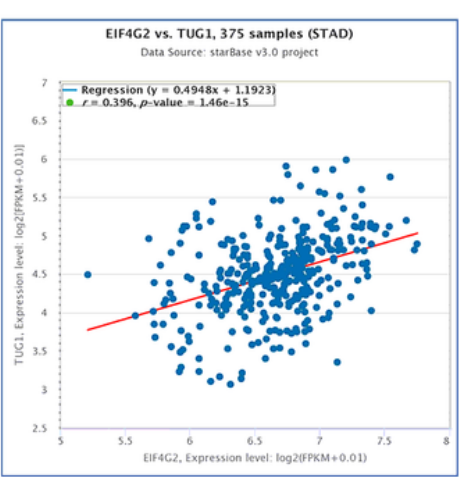

E

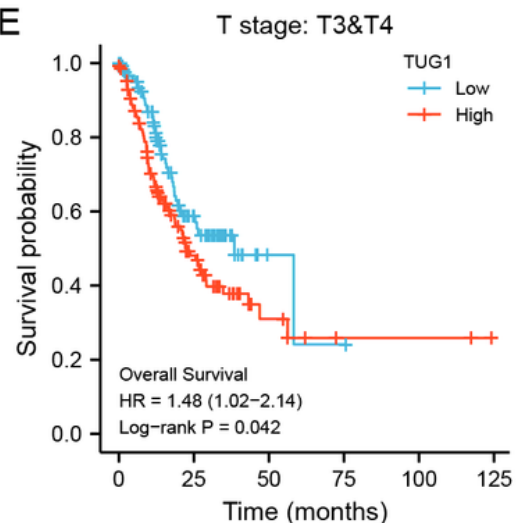

$\mathrm{H}$

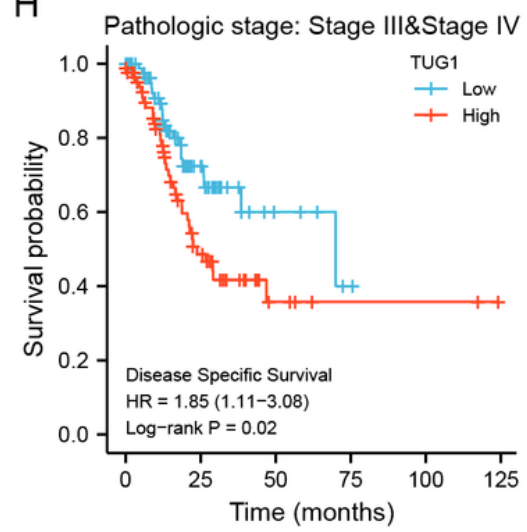

C

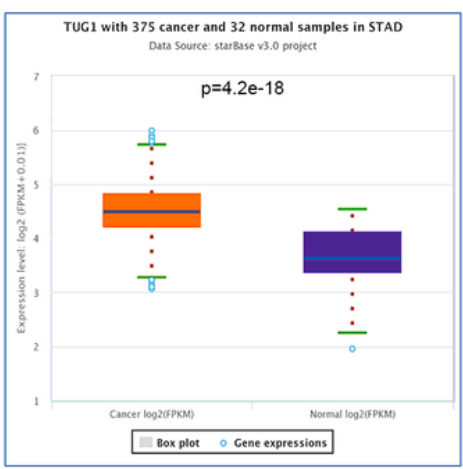

F

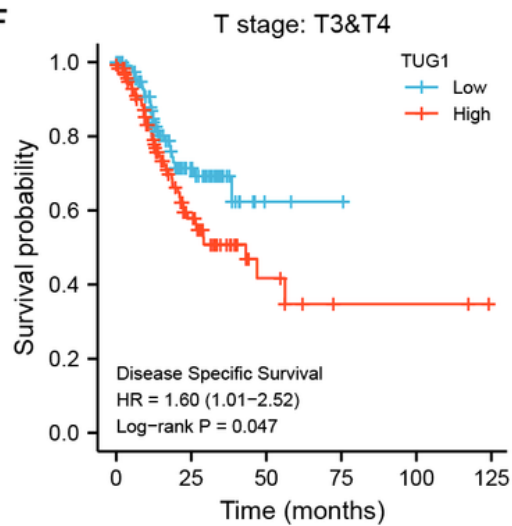

I Histologic grade: G3

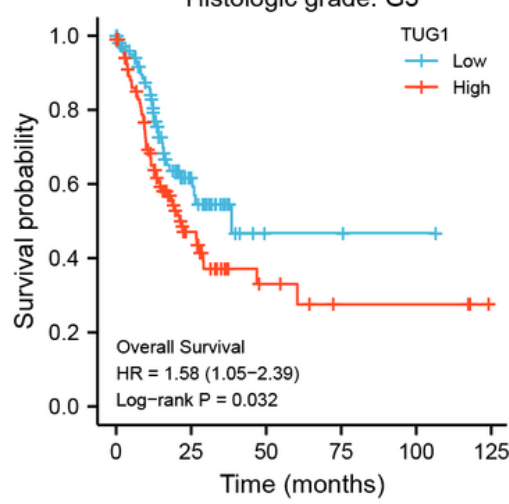

Figure 6

Analysis of TUG1 as the most likely upstream IncRNA of EIF4G2 in GC. (A) The expression correlation between hsa-miR-26a-5p and TUG1 in GC. (B) The expression correlation between EIF4G2 and TUG1 in GC. (C) The expression levels of TUG1 in 375 GC samples and 32 normal samples. (D) The expression levels of TUG1 in 27 GC and matched-adjacent normal samples. Survival curves show the association of TUG1 expression with OS and DSS in GC patients with different clinicopathological parameters: OS curve (E) and DSS curve (F) in T3 \& T4 patients, OS curve (G) and DSS curve (H) in Stage III \& Stage IV patients, OS curve (I) in grade 3 patients. ${ }^{* \star *} \mathrm{P}<0.001,{ }^{*} \mathrm{P}<0.05$. 